\title{
Simulation and Analysis of Indoor Visible Light Propagation Characteristics Based on the Method of SBR/Image
}

\author{
Liu Yuan-jian, Shi Qin-jian, Ma Xue, and Zhang Ye-rong \\ College of Electronic Science and Engineering, Nanjing University of Posts \& Telecommunications, Nanjing, Jiangsu 210003, China \\ Correspondence should be addressed to Liu Yuan-jian; liuyj@njupt.edu.cn
}

Received 30 July 2013; Accepted 22 January 2014; Published 26 February 2014

Academic Editor: Michele Midrio

Copyright ( 2014 Liu Yuan-jian et al. This is an open access article distributed under the Creative Commons Attribution License, which permits unrestricted use, distribution, and reproduction in any medium, provided the original work is properly cited.

\begin{abstract}
The indoor visible light propagation characteristics are simulated and analyzed using the method of SBR/Image (shooting and bounding ray tracing/Image). A good agreement is achieved between the results simulated and the results given in published literature. So the correctness of the method has been validated. Some propagation parameters are obtained in the simulation, such as the indoor received power distribution, statistical distribution of phase angle of received power, RMS (root mean square) delay spread, direction of arrival, and Doppler shift. The foundation for the wireless network coverage of indoor visible light communication system is provided by the analysis of the above results.
\end{abstract}

\section{Introduction}

Recently, VLC (visible light communication) has been an emerging wireless optical communication technology. LED (light emitting diode) is used in wireless base stations instead of antenna in the visible light communication system and the communication speed is up to several megabits per second. It is well known that electromagnetic interference can cause disastrous consequences in some special areas, such as space stations, airplanes, and hospitals. So the radio products are not allowed to use these particular environments. The visible light does not cause electromagnetic interference for its high frequency; thus, visible light communication technology has broad application prospects. Visible light communication theory is firstly proposed in [1]. The prototype of visible light communication technology has been presented in 1999 . Since 2000, Japan and other countries have been aware of the importance of visible light communication technology. And the indoor visible communication technology has been mainly researched. In 2000, the wireless optical communication system for wireless home link has been proposed in [2]. It has been found that the system can be used in most part of the indoor room when the data rate is $10 \mathrm{Mbps}$. In 2002, the performance characteristics of visible light communication system in the effect of the multipath due to the reflection have been analyzed and the effectiveness of the system has been shown in [3]. After 2005, the shadowing effect caused by pedestrians in the VLC has been studied in [4]. And it is shown that the system with the optimal number of the LED lighting is robust against shadowing [5]. In 2006, the intensity modulation using OFDM (orthogonal frequency division multiplexing) has been investigated [6]. The results have indicated that it is possible to cover a distance of up to one meter using a single LED. In 2007, the COFDM (coded OFDM) with QPSK (quadrature phase shift keying) modulation for the VLC has been studied in [7]. BER (bit error rate) of $2 \times 10^{-5}$ is achieved for a distance of $90 \mathrm{~cm}$ between transmitter and receiver. In 2008, the efficient bandwidth of indoor optical wireless communications has been studied in [8]. It is demonstrated that data rate of $101 \mathrm{Mbit} / \mathrm{s}$ has been achieved in experimental investigations. An example of VLC is shown in Figure 1.

Light is an electromagnetic wave, so the propagation characteristics of visible light can be researched by the methods of studying electromagnetic wave. It is particularly important to study the propagation characteristics of visible light in the indoor environment, because VLC is mainly used in office, laboratory, and so on. Wavelength of visible light is in the range of $380 \mathrm{~nm}-780 \mathrm{~nm}$, and the frequency is up to terahertz. The indoor propagation characteristics of visible 


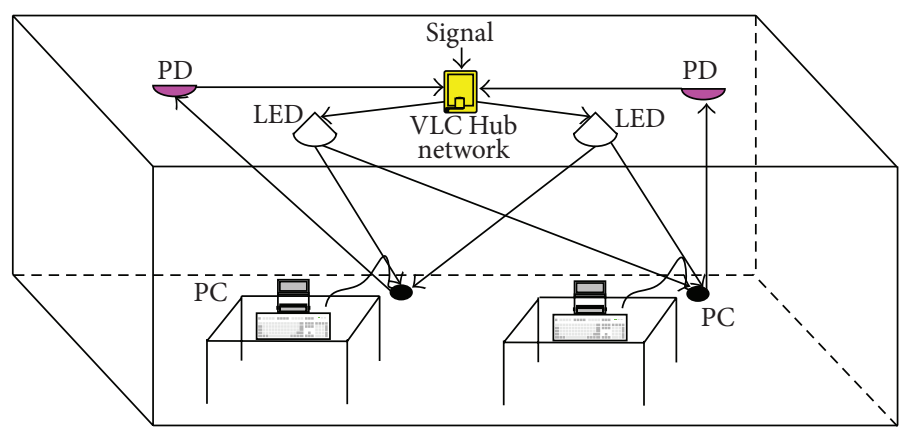

FIgURE 1: Indoor visible light communication system.

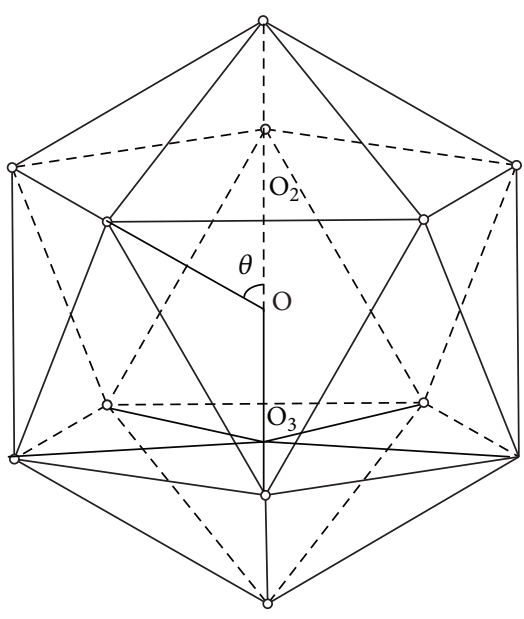

(a)

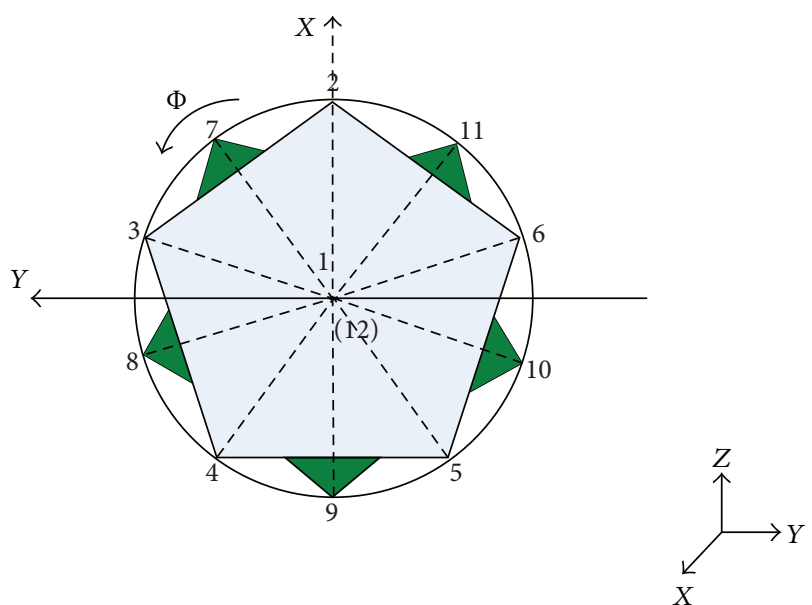

(b)

FIgURE 2: Regular icosahedron in the interior of wavefront ball.

light can be predicted by ray tracing methods. Ray tracing method includes image method, minimum optical path method, test-ray method, shooting and bounding ray tracing method, deterministic ray tube method, and shooting and bounding ray tracing/image method (SBR/Image). The image method $[9,10]$ is a simple ray tracing method because it does not need to do the intersection test. But it can only be applied to the simple structure of the environment. The minimum optical path method [11] is essentially the same as the traditional image method. And the minimum optical path to determine the location of the reflection point is used. It also cannot be applied to the complex environment. The test ray method [12] does not need to determine the location of the receiving points in advance, but they are needed to make receiving ball. It can be applied to the complex environment, but the prediction accuracy of this method is affected by the radius of receiving ball. So the calculated efficiency is not high. The shooting and bounding ray tracing method [13] does not need receiving ball, but this method takes up a lot of computer resources. The deterministic ray tube method [14] saves computer resources, but it needs to create a ray tree based on the actual environment, and it is complex. The shooting and bounding ray tracing/image method $[15,16]$ can be used for any complex propagation environment, and all the radio wave propagation paths from the transmitter to the receiver with high accuracy and computational efficiency can be found. So this method is a valuable method which can be used to predict the radio wave propagation. In this paper, the indoor visible light propagation characteristics are simulated based on the shooting and bounding ray tracing/image method.

\section{The Realization of SBR/Image}

2.1. Indoor Structure Representation. After the indoor building structure is simplified, the geometry data and the form data of each plane can be recorded while every face is given a unique identifier to represent the plane. Then data is entered into the database in accordance with the prescribed format, and the appropriate priority list will be established.

2.2. Wavefront Ball Generation. The radio beams are radiated from the transmitting antenna form many ray tubes. The wavefront ball should be divided to generate the ray tubes, and the shape and size of each wavefront are similar. The shape of wavefront can be an equilateral triangle, quadrilateral, or hexagonal. In this paper, the commonly method is used to divide the wavefront ball. A regular icosahedron in the interior of wavefront ball is shown in Figure 2, and twelve vertex coordinates of the regular icosahedron can be calculated $[17,18]$. 


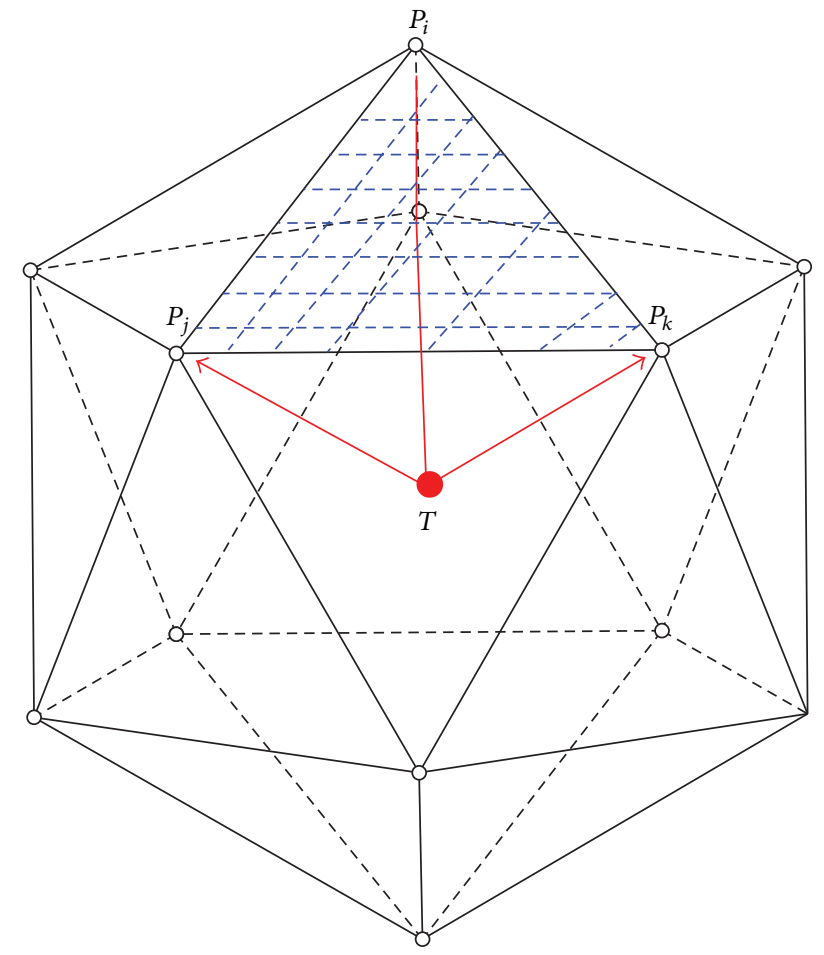

Figure 3: Establishment of the emitted ray tube.

2.3. The Establishment of the Emitted Ray Tubes. The tetrahedral ray tube with section of equilateral triangle is shown in Figure 3. The coordinate of the emission point source is $T\left(x_{t}, y_{t}, z_{t}\right)$, and the coordinate of the point of receiver is $R\left(x_{r}, y_{r}, z_{r}\right)$. The radius of wavefront ball is $r$, and twelve vertex coordinates of the regular icosahedron are $P_{n}\left(x_{n}, y_{n}, z_{n}\right)$ $(n=1,2, \ldots, 12)$.

2.4. Intersection Test. Figure 4 shows the model of intersection test. Assuming that the starting point and the end point are, respectively, denoted by $\vec{r}_{1}$ and $\vec{r}_{2}$, the arbitrary point in space will be $\vec{r}(t)=\vec{r}_{1}+t\left(\vec{r}_{2}-\vec{r}_{1}\right)$. The unit normal vector of plane which is defined as $A X+B Y+C Z+D=0$ is $\vec{n}$, and the distance of the plane from the origin of coordinate is $d=|D| / \sqrt{A^{2}+B^{2}+C^{2}}$. If the vector of ray is satisfied with the equation $\left(\vec{r}_{2}-\vec{r}_{1}\right) \cdot \vec{n}=0$, the ray is parallel to the plane. If the vector of ray is satisfied with the equation $\left(\vec{r}_{2}-\vec{r}_{1}\right) \cdot \vec{n}=0$ and $\vec{r}_{1} \cdot \vec{n}+d=0$, the ray is in the plane, so the ray and the plane have an infinite number of the intersections. If the vector of ray is satisfied with the equation $\left(\vec{r}_{2}-\vec{r}_{1}\right) \cdot \vec{n} \neq 0$, the ray and the plane have only an intersection, and the ray parameter $\left(t_{i}\right)$ at the intersection is $t_{i}=-\left(\vec{r}_{1} \cdot \vec{n}-d\right) /\left(\left(\vec{r}_{2}-\vec{r}_{1}\right) \cdot \vec{n}\right)$. If the ray parameter is satisfied with the range $0<t_{i}<1$, the ray and the plane are intersected at the point between the two end points of the ray, and the vector of intersection is satisfied with the equation $\vec{r}_{i}=\vec{r}_{1}+t_{i}\left(\vec{r}_{2}-\vec{r}_{1}\right)$ [17].

2.5. The Determination of Reflected Ray Tubes. The image source point can be located using the image method, and three intersection points of three rays of the emitted ray tube

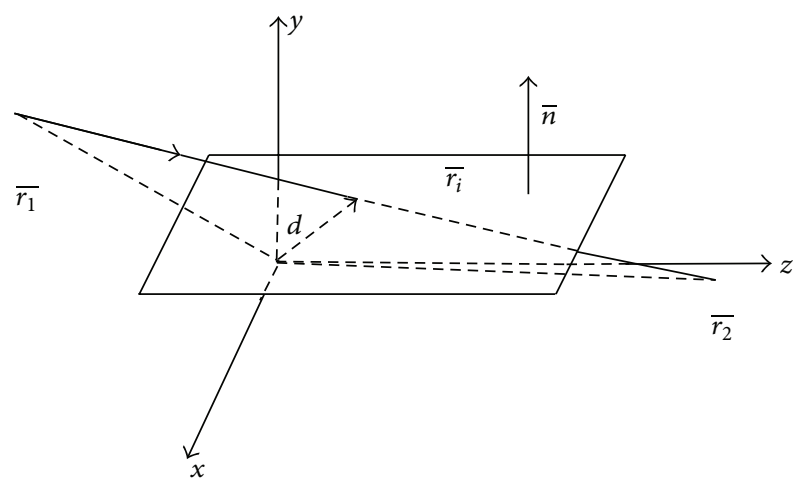

FIGURE 4: The model of intersection test.

and plane can be determined. So the reflected ray tubes will be obtained by the four points.

2.6. The Calculation of Received Field. The received field is the vector sum of the ray tubes filed which can reach the receiving point.

(1) The calculation formula of the direct field is $\vec{E}=\vec{E}_{0}$. $e^{-j k|T R|} /|T R| . \vec{E}_{0}$ is the electric filed value of ray tube $T-P_{i} P_{j} P_{k}$ in the reference position $\Delta P_{i} P_{j} P_{k}$ and $k$ is the wave number. $|T R|$ is the actual propagation distance when the ray tube $T-P_{i} P_{j} P_{k}$ reaches received point $R$.

(2) The calculation formula of the reflected field is $\vec{E}=$ $\vec{E}_{0} \cdot\left\{\Pi \overline{\overline{R_{i}}}\right\} \cdot\left\{\Pi \overline{\overline{T_{i}}}\right\} \cdot\left\{\Pi e^{-\gamma_{i} l_{i}}\right\} .\left\{\Pi \overline{\overline{R_{i}}}\right\}$ is the dyad of the reflection coefficient:

$$
\begin{gathered}
\overline{\bar{R}}_{i}=R_{i_{\perp}} \bar{e}_{\perp} \bar{e}_{\perp}+R_{i \|} \bar{e}_{\|} \bar{e}_{\|}, \\
R_{i \perp}=\frac{\cos \theta_{i}-\sqrt{\varepsilon_{2} / \varepsilon_{1}-\sin ^{2} \theta_{i}}}{\cos \theta_{i}+\sqrt{\varepsilon_{2} / \varepsilon_{1}-\sin ^{2} \theta_{i}}} \\
R_{i \|}=\frac{\left(\varepsilon_{2} / \varepsilon_{1}\right) \cos \theta_{i}-\sqrt{\varepsilon_{2} / \varepsilon_{1}-\sin ^{2} \theta_{i}}}{\left(\varepsilon_{2} / \varepsilon_{1}\right) \cos \theta_{i}+\sqrt{\varepsilon_{2} / \varepsilon_{1}-\sin ^{2} \theta_{i}}} .
\end{gathered}
$$

$\left\{\Pi \overline{\overline{T_{i}}}\right\}$ is the dyad of the transmission coefficient: $\overline{\overline{T_{i}}}=$ $T_{i_{\perp}} \bar{e}_{\perp} \bar{e}_{\perp}+T_{i \|} \bar{e}_{\|} \bar{e}_{\|}, T_{i_{\perp}}=R_{i_{\perp}}+1$, and $T_{i \|}=\left(R_{i \|}+1\right)$. $\sqrt{\varepsilon_{1} / \varepsilon_{2}} \cdot \gamma_{i}$ is the change of phase relative to the reference position $\Delta P_{i} P_{j} P_{k}$ and the $l_{i}$ is the change of attenuation relative to the reference position.

\section{Numerical Results}

A room environment appeared in the literature [19] is chosen for our simulation. This room with dimensions $5 \mathrm{~m} \times 5 \mathrm{~m} \times$ $2.5 \mathrm{~m}$ is shown in Figure 5, in which its ceiling, floor, and walls are all made of a pure dielectric with $\varepsilon_{r}=4$ [20]. The LED lightings are installed at a height of $2.5 \mathrm{~m}$ from the floor. The number of the LED lighting equipments is 4 , and each 


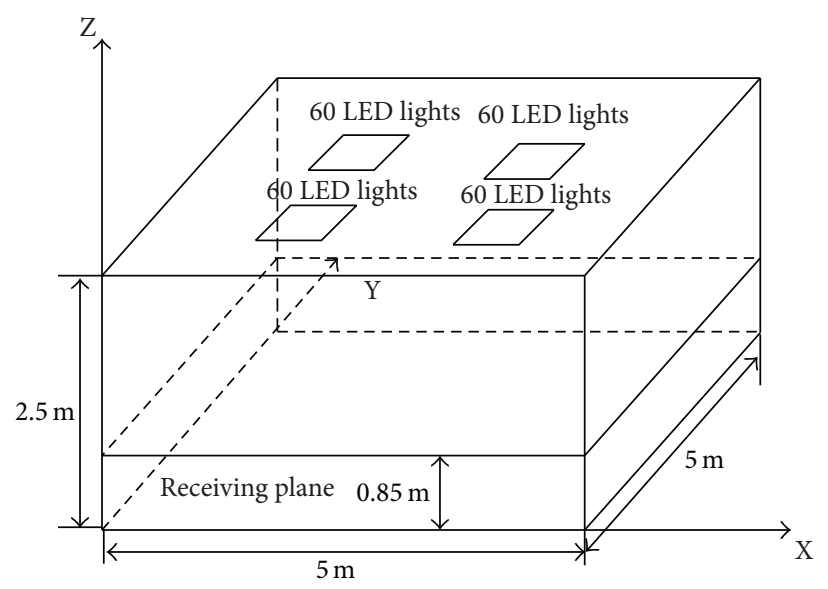

(a)

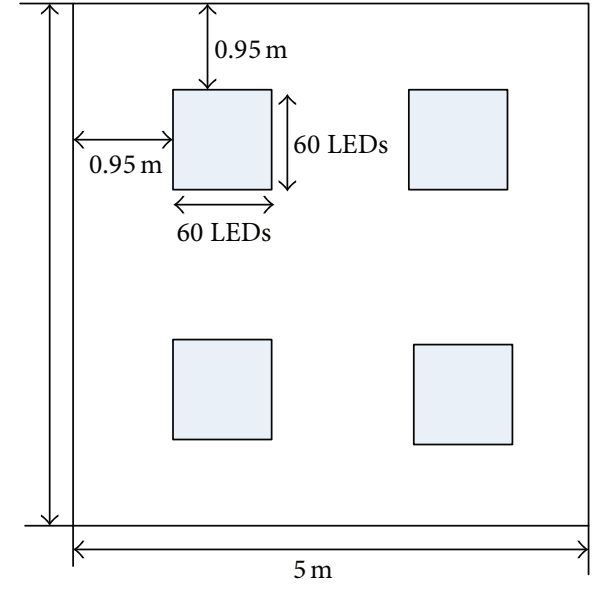

(b)

FIGURE 5: Simulation environment: (a) 3D Schematic and (b) 2D Schematic.

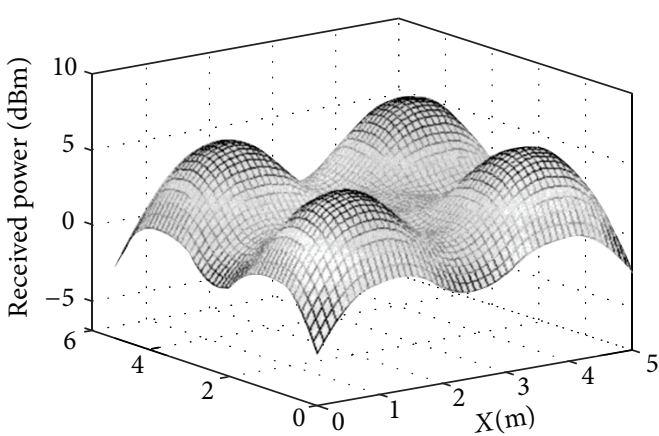

(a)

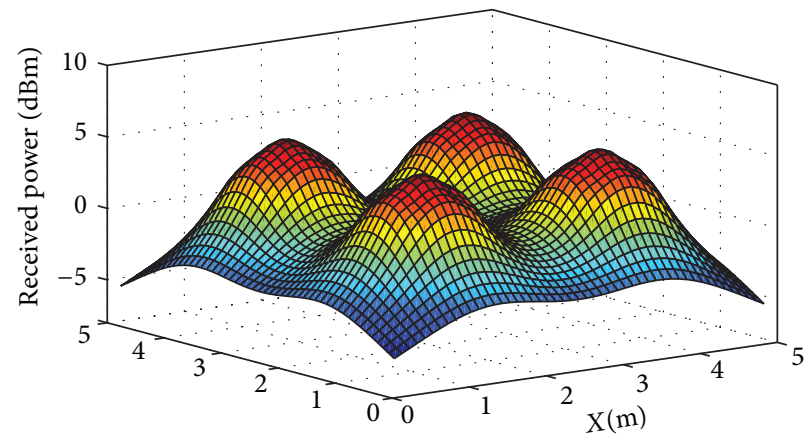

(b)

FIGURE 6: Received power (a) results in literature [19] and (b) simulated result.

LED lighting equipment is filled with $3600(60 \times 60)$ LEDs. The semiangle at half-power of an LED is $60 \mathrm{deg}$, and the transmitted optical power of an LED is $20 \mathrm{~mW}$. The height of receiving plane is $0.85 \mathrm{~m}$.

3.1. Correctness Verification. The received power in the literature [19] varies between $-3.85 \mathrm{dBm}$ and $6.22 \mathrm{dBm}$ as shown in Figure 6(a). And the simulated result varies between $-3.36 \mathrm{dBm}$ and $7.46 \mathrm{dBm}$ as shown in Figure 6(b). Moreover, the comparison of received power distribution between simulated result and measured result in the literature [19] shows a good agreement as indicated in Figure 6. So the correctness of SBR/Image has been validated. The following is the reasons for the subtle difference between the simulated result and result in literature. (1) The electromagnetic characteristics between the simulation and actual material are different. (2) The number of received points in literature is less than that in this simulation.

3.2. The Distribution of Indoor Received Power. The statistical distribution of phase angle of received power which obeys uniform distribution is demonstrated in Figure 7. The received power rate of each path (direct path, single reflected
TABLE 1: The received power rate of each path.

\begin{tabular}{lc}
\hline & Received power rate \\
\hline Direct path & $95.81 \%$ \\
Single reflected path & $3.24 \%$ \\
Double reflected path & $0.83 \%$ \\
Trinal reflected path & $0.12 \%$ \\
\hline
\end{tabular}

path, double reflected path, and trinal reflected path) is shown in Table 1. The received power rate of the double reflected path and the trinal reflected path is small enough compared with the direct path and the single reflected path.

3.3. The Root Mean Square (RMS) Delay Spread. The RMS delay spread of visible light in an indoor environment is given in Figure 8. The RMS delay spread is the second moment of power delay profile, and it is defined as

$$
\sigma=\sqrt{\frac{\sum_{i=1}^{N_{p}} P_{i} t_{i}^{2}}{P_{R}}-\bar{t}^{2}},
$$




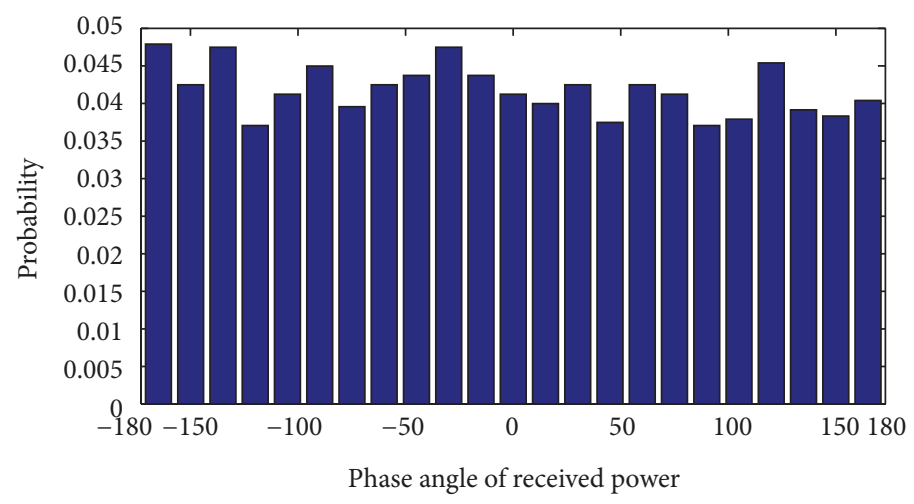

FIgURE 7: The phase angle of received power.

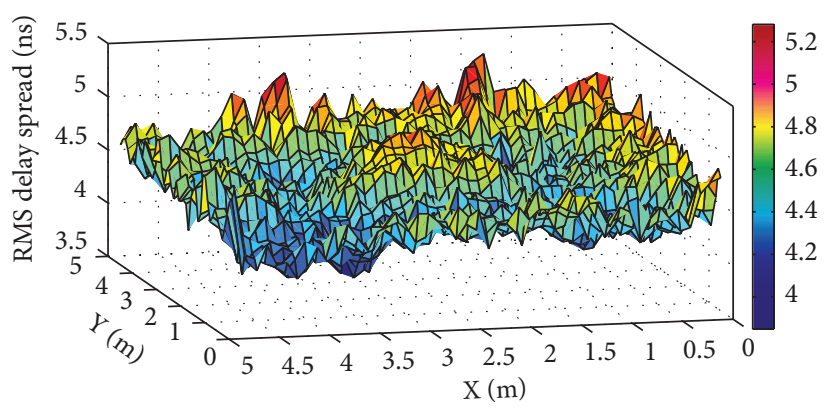

(a)

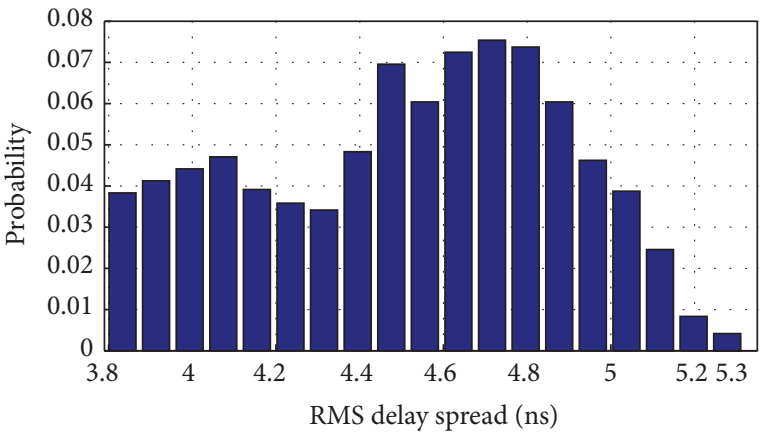

(b)

FIGURE 8: The RMS delay spread.

where $\bar{t}$ is the average time of arrival, and it is defined as $\bar{t}=$ $\left(\sum_{i=1}^{N_{p}} P_{i} t_{i}\right) / P_{R} . P_{i}$ is the received power of the $i$ th path. $P_{R}$ is the total of received power. The RMS delay spread is a better measurement for the spread of multipath, and it varies in the level of ns.

In this paper, the RMS delay spread of visible light varies between $3.8 \mathrm{~ns}$ and $5.3 \mathrm{~ns}$ as shown in Figure 8. The worst RMS delay spread occurs at the edge of the room, and the least RMS delay spread occurs at the place where it is just below the LED. This is because when the receiver point is just below the LED, there are almost only reflected paths from surface of the floor and ceiling besides the direct path. The RMS delay spread of visible light is small compared with the typical wireless communications bands such as $900 \mathrm{MHz}$ and $2.4 \mathrm{GHz}$.

3.4. The Direction of Arrival. The direction of arrival which is defined as $\bar{a}=\sin \left(\theta_{A}\right) \cos \left(\phi_{A}\right) \bar{x}+\sin \left(\theta_{A}\right) \sin \left(\phi_{A}\right) \bar{y}+\cos \left(\phi_{A}\right) \bar{z}$ can be obtained.

Figure 9 shows the mean angle of arrival of all the received points. The $\bar{\theta}$ is defined as $\bar{\theta}=\tan ^{-1}\left(\sqrt{A_{x}^{2}+A_{y}^{2}} / A_{z}\right)$, and the $\bar{\phi}$ is defined as $\bar{\phi}=\cos ^{-1}\left(A_{y} / A_{x}\right)\left(A=\sum_{i=1}^{N} P_{i} \bar{a}\right)$. The mean angle of arrival $(\bar{\theta})$ varies between $63^{\circ}$ and $77^{\circ}$. The mean angle of arrival $(\bar{\phi})$ varies between $20^{\circ}$ and $350^{\circ}$.
3.5. Doppler Shift. The formula of Doppler shift is $\Delta f=$ $f_{0}\left[\left(\bar{d} \cdot \overline{V_{T}}+\bar{a} \cdot \overline{V_{R}}\right) / c\right]$. So each path will cause Doppler shift if the transmitter or the receiver is moving fast. The effect of Doppler shift must be considered. So Doppler shift can be determined (assuming that the transmitter is fixed and the speed of receiver is $1 \mathrm{~m} / \mathrm{s}$ ). The Doppler shift varies between $-1.3 \mathrm{MHz}$ and 1.3 MHz as shown in Figure 10. This simulation environment is similar to indoor mobile communication environment, so the theoretical foundation for the indoor coverage of visible light communication system is provided by the range of Doppler shift in this simulation.

\section{Conclusions}

In this paper, the indoor visible light propagation characteristics are simulated and analyzed by using the method of SBR/Image. And a good agreement is acquired in the comparison between simulated results and results given in some published literatures, so the correctness of the method has been validated. The received power rate of double reflected path and the trinal reflected path is small enough compared with the direct path and the single reflected path. And the statistical distribution of phase angle of received power is subject to the uniform distribution. The RMS delay spread of indoor visible light varies between $3.8 \mathrm{~ns}$ and $5.3 \mathrm{~ns}$ in this simulation which is small compared with 

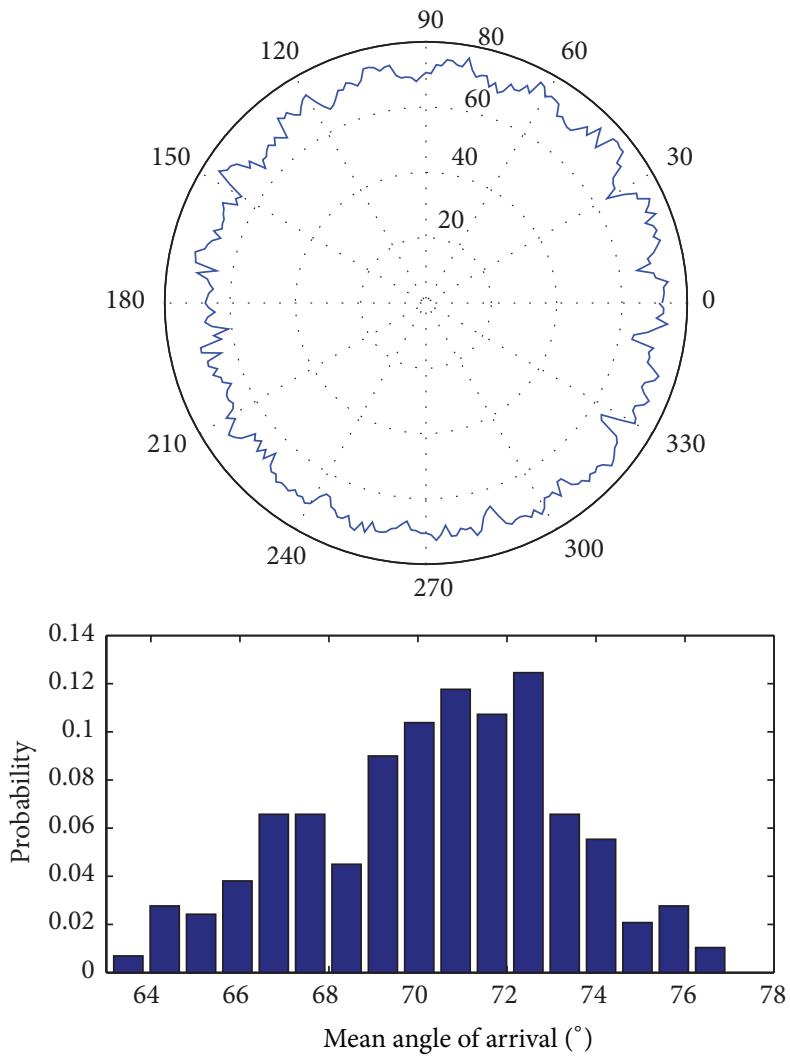

(a) Mean angle of arrival $\bar{\theta}$
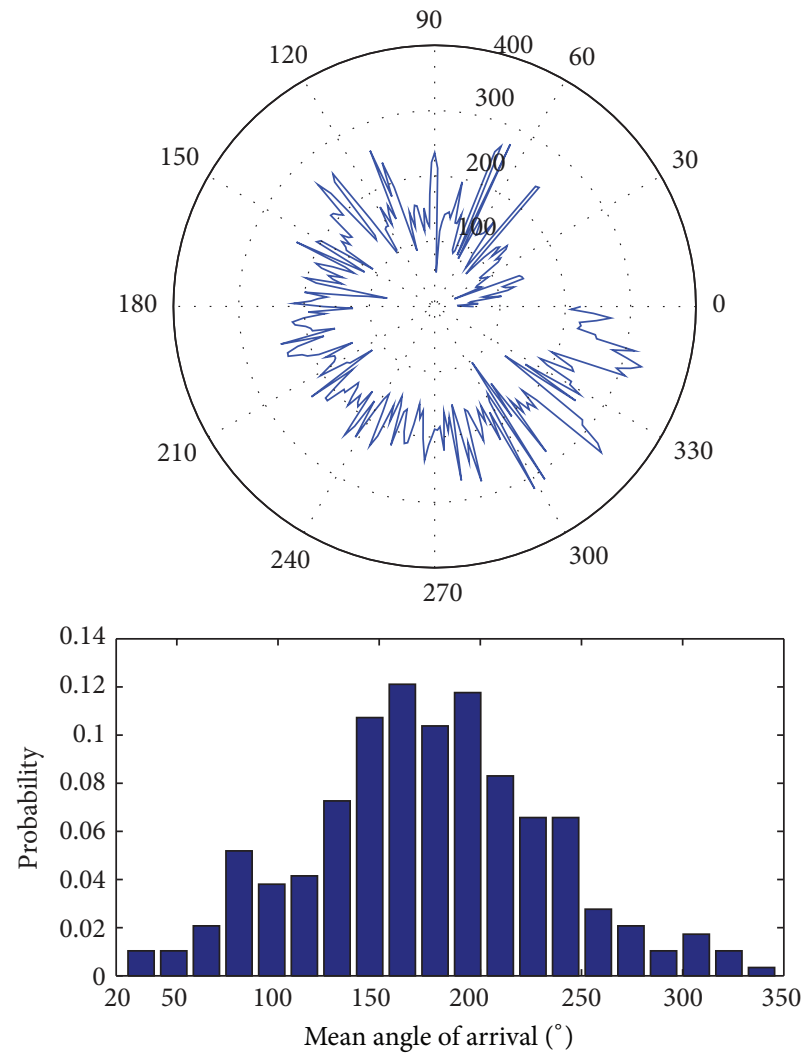

(b) Mean angle of arrival $\bar{\phi}$

FIGURE 9: Mean angle of arrival.

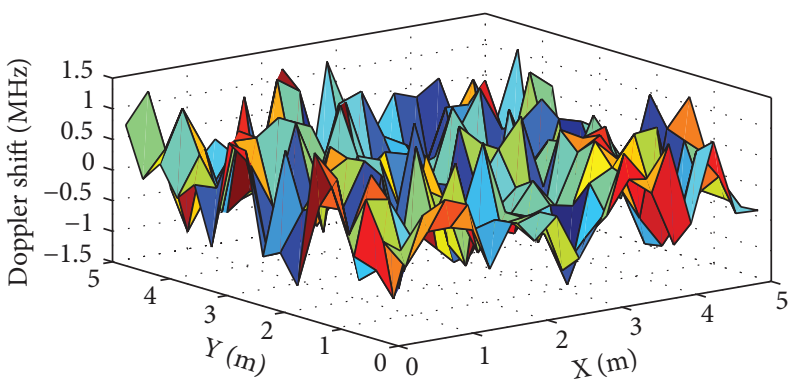

(a)

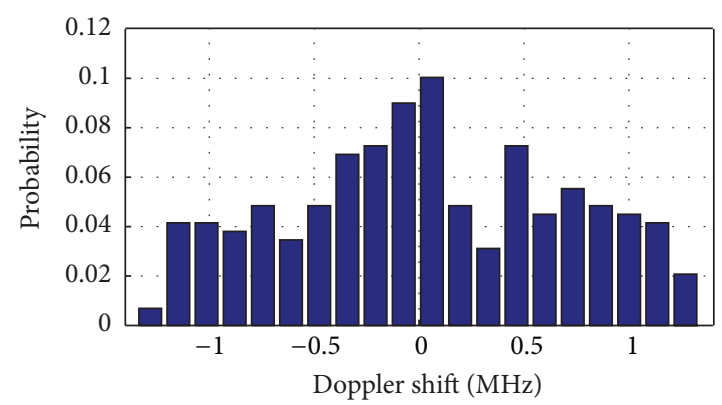

(b)

FIgURE 10: Doppler shift.

the typical wireless communications bands such as $900 \mathrm{MHz}$ and $2.4 \mathrm{GHz}$. Assuming that the transmitter is fixed and the speed of receiver is $1 \mathrm{~m} / \mathrm{s}$, the Doppler shift will vary between $-1.3 \mathrm{MHz}$ and $1.3 \mathrm{MHz}$ which is large compared with the typical wireless communication bands. The foundation for the wireless network coverage of indoor visible light communication system is provided by the analysis of the above results. This simulation environment is almost an empty room. However, the actual indoor environment is usually not so simple for the simulation. Further research of indoor visible light propagation characteristics based on SBR/Image in complex indoor environments would be required, which is our next research direction.

\section{Conflict of Interests}

The authors declare that there is no conflict of interests regarding the publication of this paper.

\section{Acknowledgments}

This work has been supported by the National Natural Science Fund under Grant no. 61372045 and by the Ministry of 
Education of Higher Specialized Research Fund for the Doctoral Program under Grant no. 20123223120003.

\section{References}

[1] G. Pang, K.-L. Ho, T. Kwan, and E. Yang, "Visible light communication for audio systems," IEEE Transactions on Consumer Electronics, vol. 45, no. 4, pp. 1112-1118, 1999.

[2] Y. Tanaka, S. Haruyama, and M. Nakagawa, "Wireless optical trasnsmissions with white colored LED for wireless home links," in Proceedings of the 11th IEEE International Symposium on Personal, Indoor and Mobile Radio Communications (PIMRC '00), pp. 1325-1329, September 2000.

[3] K. Fan and T. Komine, "The effect of reflection on indoor visible light communication system utilizing white LEDs," in Proceedings of the 5th International Symposium on wireless Personal Multimedia Communications, vol. 3, pp. 611-615, 2002.

[4] T. Komine, S. Haruyama, and M. Nakagawa, "A study of shadowing on indoor visible-light wireless communication utilizing plural white LED lightings," Wireless Personal Communications, vol. 34, no. 1-2, pp. 211-225, 2005.

[5] T. Komine, J. H. Lee, S. Haruyama, and M. Nakagawa, "Adaptive equalization system for visible light wireless communication utilizing multiple white led lighting equipment," IEEE Transactions on Wireless Communications, vol. 8, no. 6, pp. 2892-2900, 2009.

[6] M. Z. Afgani, H. Haas, H. Elgala, and D. Knipp, "Visible light communication using OFDM," in Proceedings of the 2nd International Conference on Testbeds and Research Infrastructures for the Development of Networks and Communities (TRIDENTCOM '06), pp. 129-134, March 2006.

[7] H. Elgala, R. Mesleh, H. Haas, and B. Pricope, "OFDM visible light wireless communication based on white LEDs," in Proceedings of the IEEE 65th Vehicular Technology Conference (VTC '07), pp. 2185-2189, April 2007.

[8] J. Grubor, S. Randel, K.-D. Langer, and J. W. Walewski, "Bandwidth-efficient indoor optical wireless communications with white light-emitting diodes," in Proceedings of the 6th International Symposium Communication Systems, Networks and Digital Signal Processing (CSNDSP 08), pp. 165-169, July 2008.

[9] S. Y. Tan and H. S. Tan, "A microcellular communications propagation model based on the uniform theory of diffraction and multiple image theory," IEEE Transactions on Antennas and Propagation, vol. 44, no. 10, pp. 1317-1326, 1996.

[10] S. Grubisic, W. P. Carpes Jr., C. B. Lima, and P. Kuo-Peng, "Raytracing propagation model using image theory with a new accurate approximation for transmitted rays through walls," IEEE Transactions on Magnetics, vol. 42, no. 4, pp. 835-838, 2006.

[11] M. G. Sánchez, L. De Haro, A. G. Pino, and M. Calvo, "Exhaustive ray tracing algorithm for microcellular propagation prediction models," Electronics Letters, vol. 32, no. 7, pp. 624-625, 1996.

[12] W. Honcharenko, H. L. Bertoni, J. L. Dailing, J. Qian, and H. D. Yee, "Mechanisms governing UHF propagation on single floors in modern office buildings," IEEE Transactions on Vehicular Technology, vol. 41, no. 4, pp. 496-504, 1992.

[13] H. Shirai, R. Sato, and K. Otoi, "Electromagnetic wave propagation estimation by 3-D SBR method," in Proceedings of the International Conference on Electromagnetics in Advanced Applications (ICEAA '07), pp. 129-132, September 2007.

[14] H.-W. Son and N.-H. Myung, "A deterministic ray tube method for microcellular wave propagation prediction model," IEEE
Transactions on Antennas and Propagation, vol. 47, no. 8, pp. 1344-1350, 1999.

[15] S.-H. Chen and S.-K. Jeng, "SBR image approach for radio wave propagation in tunnels with and without traffic," IEEE Transactions on Vehicular Technology, vol. 45, no. 3, pp. 570-578, 1996.

[16] S.-H. Chen and S.-K. Jeng, "An SBR/image approach for radio wave propagation in indoor environments with metallic furniture," IEEE Transactions on Antennas and Propagation, vol. 45, no. 1, pp. 98-106, 1997.

[17] Z. Z. Wu, "The wireless radio propagation of mobile communication," The Publishing Company of People Posts and Telecommunications, pp. 258-261, 2002.

[18] Y.-J. Liu, H. Chen, Y.-R. Zhang, and W. Cao, "Design of software predicting indoor microcell field strength," Journal of Nanjing University of Posts and Telecommunications, vol. 27, no. 5, pp. $1-5,2007$.

[19] Y. B. Wang, Research on Indoor Visible Light Wireless Communication System, The Master Degree Dissertation of Zhejiang University, Hangzhou, China, 2008.

[20] A. M. Hammoudeh and G. Allen, "Millimetric wavelengths radiowave propagation for line-of-sight indoor microcellular mobile communications," IEEE Transactions on Vehicular Technology, vol. 44, no. 3, pp. 449-460, 1995. 

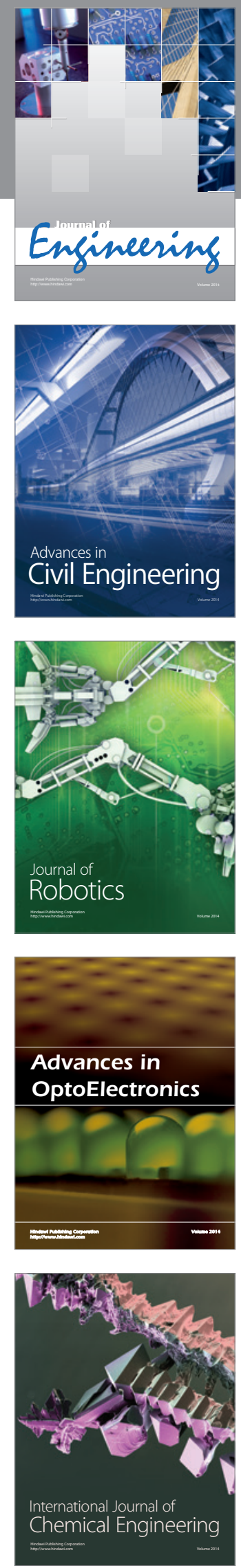

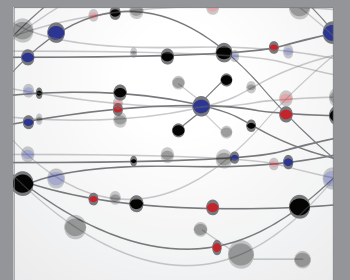

The Scientific World Journal
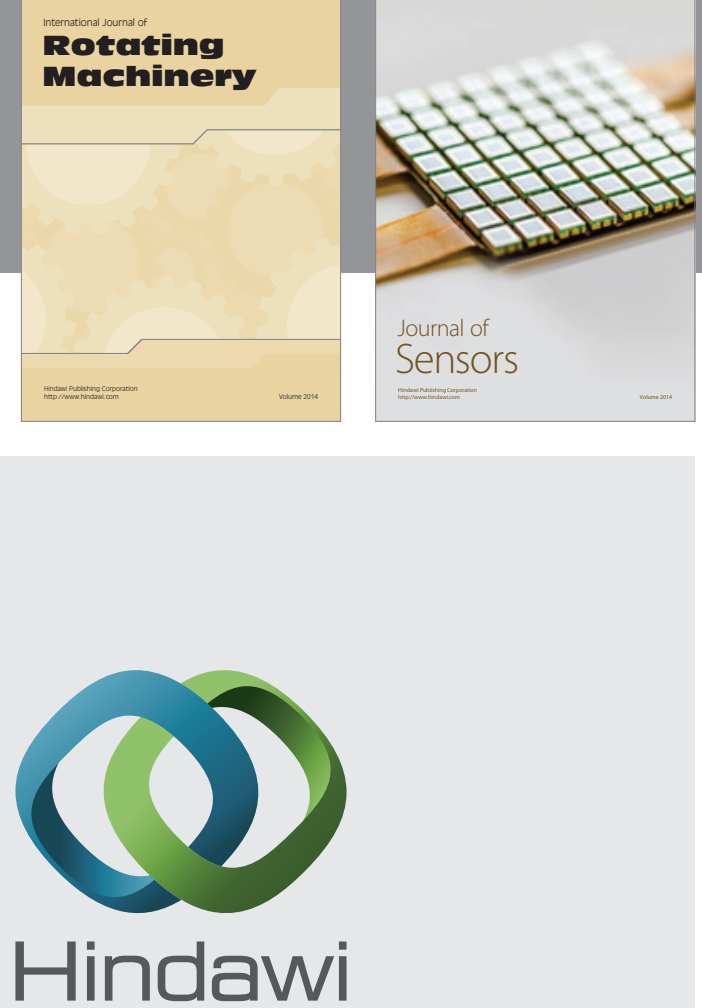

Submit your manuscripts at http://www.hindawi.com
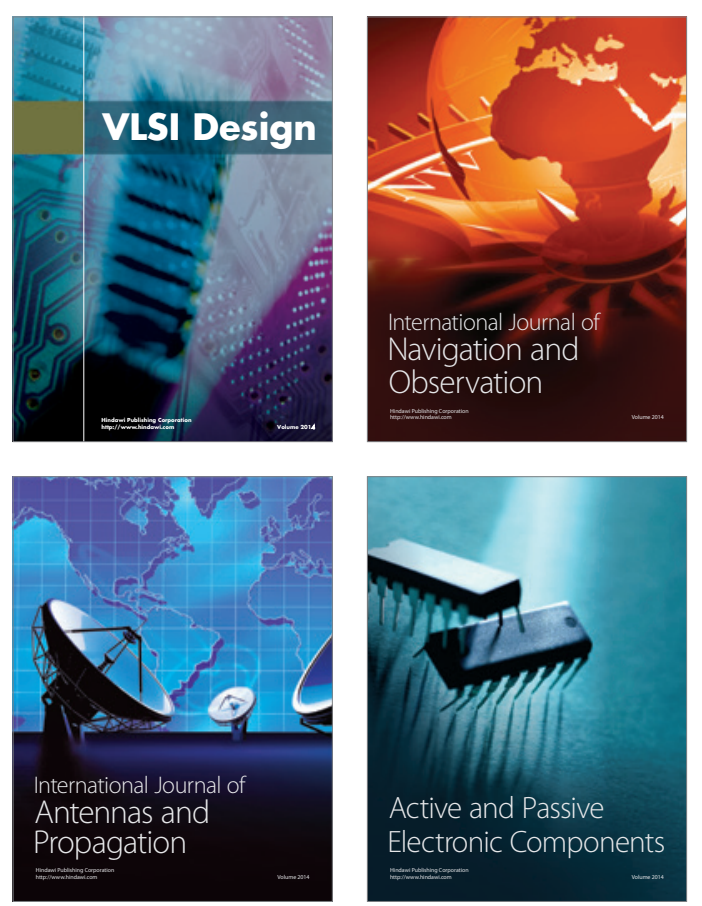
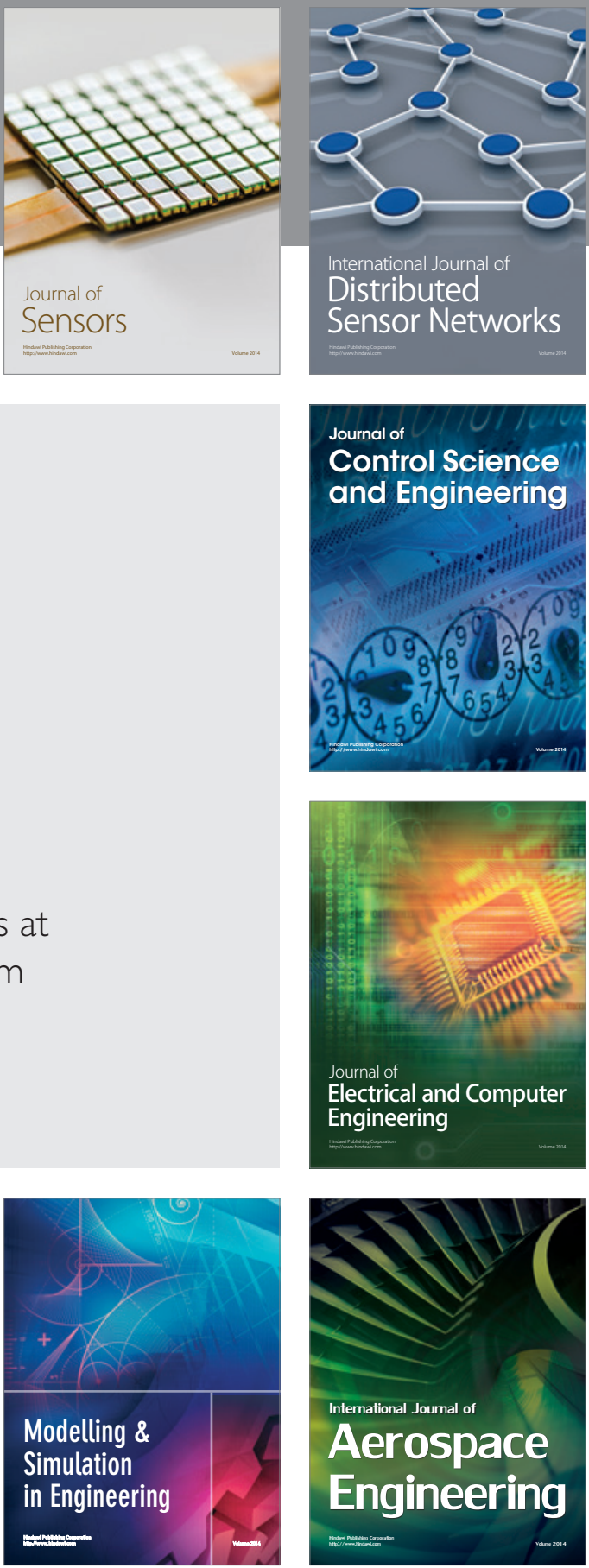

Journal of

Control Science

and Engineering
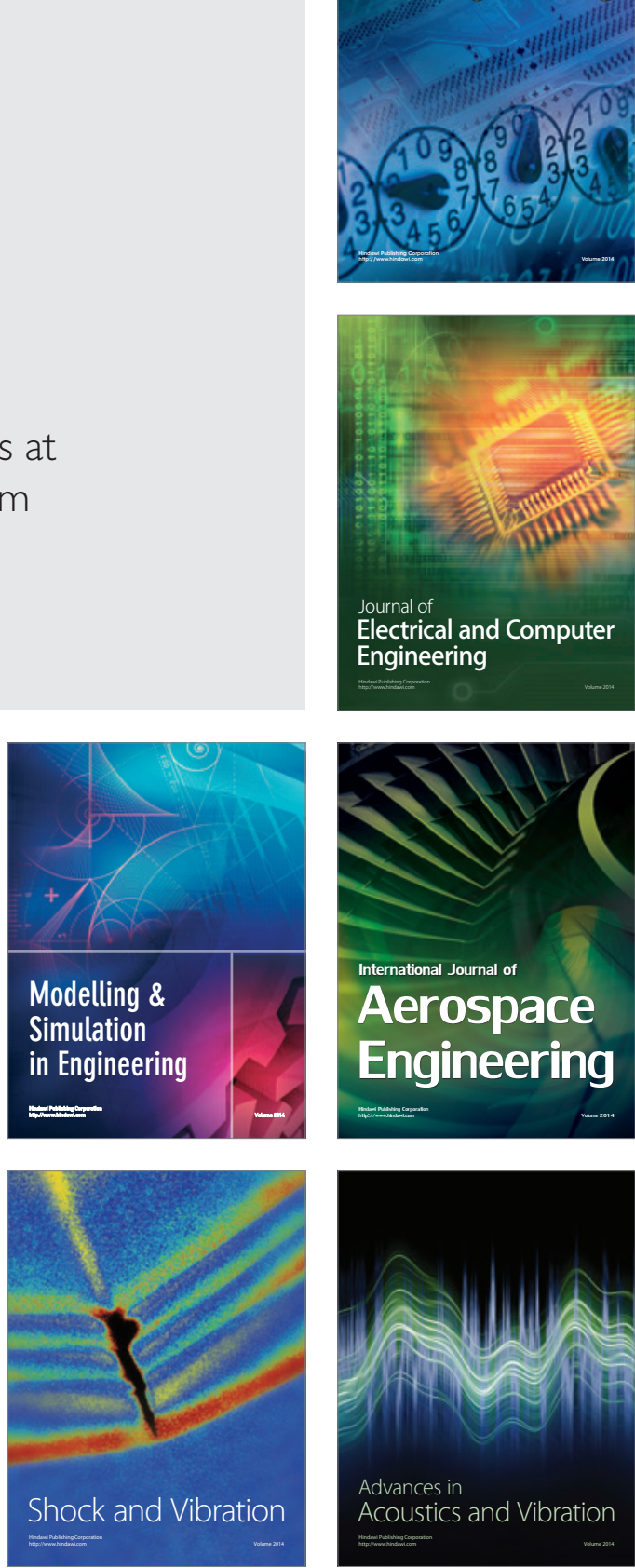